\title{
Generalized laws of refraction and reflection at interfaces between different photonic artificial gauge fields
}

Moshe-Ishay Cohen $\mathbb{1}^{1,2}$, Christina Jörg $\mathbb{1}^{3,4}$, Yaakov Lumer ${ }^{1,2}$, Yonatan Plotnik ${ }^{1,2}$, Erik H. Waller ${ }^{3}$, Julian Schulz ${ }^{3}$, Georg von Freymann $\mathbb{B}^{3,5}$ and Mordechai Segev ${ }^{1,2}$

\begin{abstract}
Artificial gauge fields the control over the dynamics of uncharged particles by engineering the potential landscape such that the particles behave as if effective external fields are acting on them. Recent years have witnessed a growing interest in artificial gauge fields generated either by the geometry or by time-dependent modulation, as they have been enablers of topological phenomena and synthetic dimensions in many physical settings, e.g., photonics, cold atoms, and acoustic waves. Here, we formulate and experimentally demonstrate the generalized laws of refraction and reflection at an interface between two regions with different artificial gauge fields. We use the symmetries in the system to obtain the generalized Snell law for such a gauge interface and solve for reflection and transmission. We identify total internal reflection (TIR) and complete transmission and demonstrate the concept in experiments. In addition, we calculate the artificial magnetic flux at the interface of two regions with different artificial gauge fields and present a method to concatenate several gauge interfaces. As an example, we propose a scheme to make a gauge imaging system - a device that can reconstruct (image) the shape of an arbitrary wavepacket launched from a certain position to a predesigned location.
\end{abstract}

\section{Introduction}

Snell's law and the Fresnel coefficients are the cornerstones of describing the evolution of electromagnetic waves at an interface between two different media. By cascading several such systems, each with its own optical properties, it is possible to design complex structures that give rise to various important devices and systems, such as lenses, waveguides ${ }^{1}$, resonators, photonic crystals $^{2}$, and even localization phenomena, when random interfaces are involved $^{3}$. The behavior of waves in the presence of an interface can exhibit fundamental features, e.g., total internal reflection (TIR), back-refraction for negative-

\footnotetext{
Correspondence: Mordechai Segev (msegev@technion.ac.il)

${ }^{1}$ Physics Department, Technion-Israel Institute of Technology, Haifa 32000, Israel

${ }^{2}$ Solid State Institute, Technion-Israel Institute of Technology, Haifa 32000

Israel

Full list of author information is available at the end of the article

These authors contributed equally: Moshe-Ishay Cohen, Christina Jörg
}

positive refraction index interfaces ${ }^{4,5}$, and even confinement of states to the interface itself, such as Tamm and Shockley states $^{6,7}$, plasmon polaritons ${ }^{8,9}$, Dyakonov states $^{10,11}$ and topological edge states ${ }^{12-14}$. Traditionally, the Fresnel equations describe the reflection and transmission of electromagnetic waves at an interface separating two media with different optical properties. These can be two materials with different permittivities or two different periodic systems (photonic crystals) composed of the same material, e.g., an interface between two dissimilar waveguide arrays ${ }^{15}$. However, an interface can also separate two optical systems that differ only by the artificial gauge fields created in them. Generally, such a "gauge interface" marks a different dispersion curve on either side of the interface; hence, it must affect the transmission and reflection at the interface.

Gauge fields (GFs) are a basic concept in physics describing forces applied on charged particles. Artificial

\section{(c) The Author(s) 2020}

(c) (i) Open Access This article is licensed under a Creative Commons Attribution 4.0 International License, which permits use, sharing, adaptation, distribution and reproduction cc) in any medium or format, as long as you give appropriate credit to the original author(s) and the source, provide a link to the Creative Commons license, and indicate if changes were made. The images or other third party material in this article are included in the article's Creative Commons license, unless indicated otherwise in a credit line to the material. If material is not included in the article's Creative Commons license and your intended use is not permitted by statutory regulation or exceeds the permitted use, you will need to obtain permission directly from the copyright holder. To view a copy of this license, visit http://creativecommons.org/licenses/by/4.0/. 

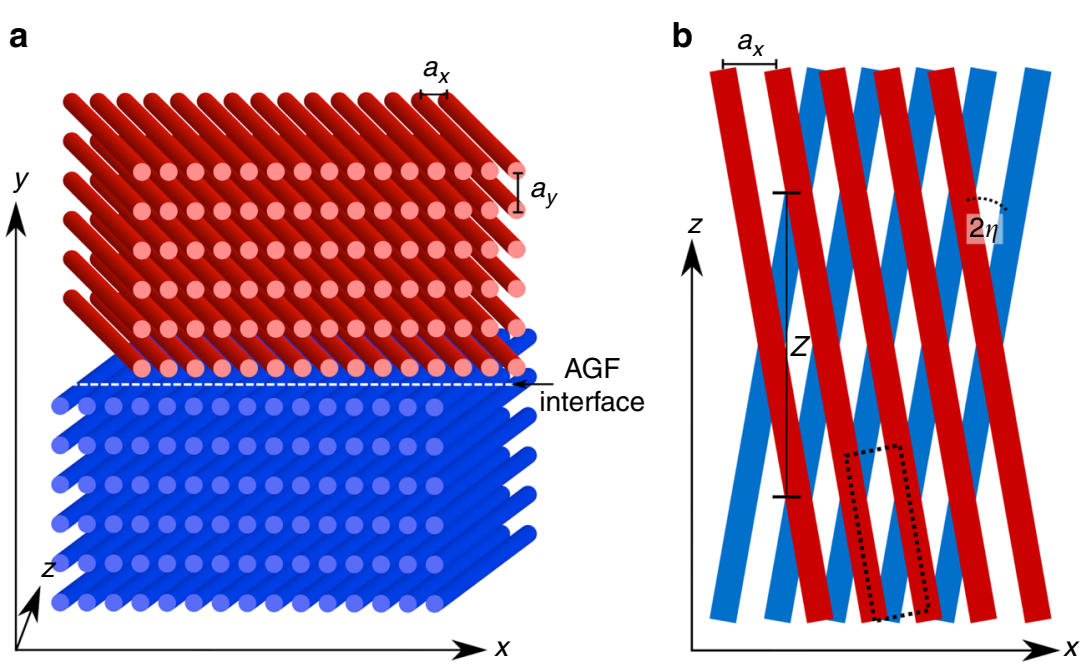

C

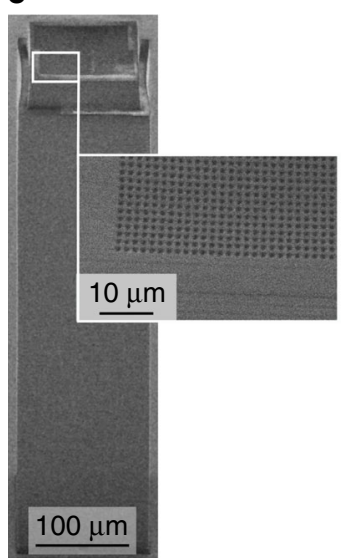

d

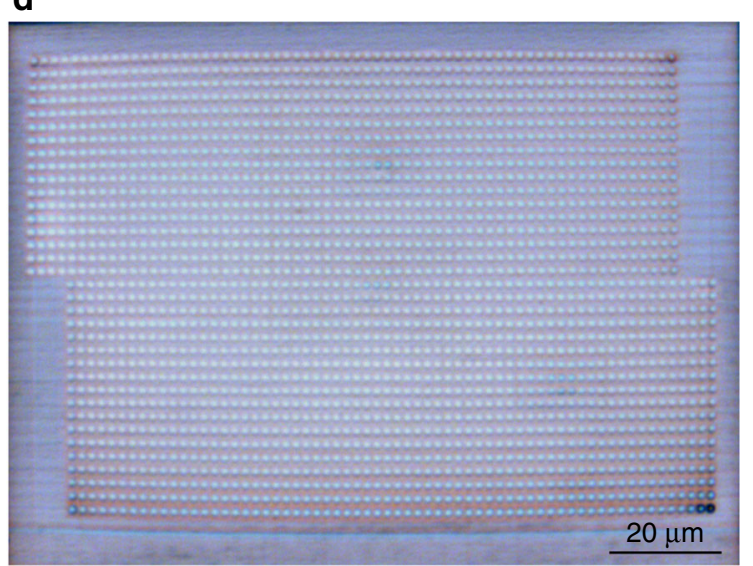

Fig. 1 Sketch of our artificial gauge interface. Two rectangular arrays of waveguides (red: upper array, blue: lower array) are stacked on top of each other, creating an artificial GF interface in the $y$-direction. The waveguide arrays are tilted by $2 \eta$ with respect to each other. Otherwise, the parameters for the two arrays are identical. a Front view. b Top view. The dashed box represents one unit cell in the $x$ - $z$-plane in the upper array. c SEM image of the inverse fabricated waveguide sample from the side. The inset shows a magnified region to visualize the hollow waveguides. $\mathbf{d}$ Microscope image of the infiltrated sample from the top

GFs are a technique for engineering the potential landscape such that neutral particles will mimic the dynamics of charged particles driven by external fields. With the advent of the particle-wave duality, artificial GFs have been demonstrated to act on photons ${ }^{16-19}$, cold atoms ${ }^{20,21}$, acoustic waves, etc. These artificial GFs are generated either by the geometry ${ }^{17}$ or by time-dependent modulation ${ }^{18}$ of system parameters. With the growing interest in topological systems ${ }^{22}$, which necessitate $\mathrm{GFs}^{23,24}$, it was suggested that the interface between two regions of the same medium but with different GFs in each region can create an effective edge. In these systems, both sides of the interface have the same basic dispersion properties, altered only by applying a different GF on each side. Such a gauge edge was employed to demonstrate analogies to the Rashba effect ${ }^{25}$, optical waveguiding ${ }^{26,27}$, topological edge states ${ }^{28,29}$ and back-refraction ${ }^{30}$. In the presence of a different GF on either side of the interface, the trajectories of waves crossing from one side to the other are governed by the symmetries in the system, which are expected to result in an effective Snell's law, whereas the reflection and transmission coefficients arise from the specific boundary.

Here, we theoretically and experimentally demonstrate the effective Snell law governing the reflection and transmission of waves at an interface between regions of the same photonic medium, differing only in the artificial gauge fields introduced on either side. We show how the transverse momenta of the reflected and transmitted waves change according to the interfacial change in the gauge field, and demonstrate TIR and complete transmission. Subsequently, we provide an approximate 
calculation for the Fresnel coefficients for our example and explain how to generalize the concepts. Finally, we show how to concatenate several "gauge interfaces" and propose a design for a gauge-based imaging system-a device composed of several interfaces between different gauge fields, acting to reconstruct (image) an arbitrary paraxial input wavepacket at a predetermined plane.

\section{Results}

For simplicity, consider first a simple system constituting an artificial gauge interface: two 2D arrays of evanescently coupled waveguides, where the waveguides in each array follow a different trajectory along the propagation axis $z$ (Fig. 1). This model system serves to explain the ideas involved, which are later generalized. The GFs in our system are a direct result of the trajectories of the waveguides and do not require any temporal modulation of the materials at hand. The upper array experiences a constant tilt in the $x$-direction from the propagation axis $z$, with a paraxial angle $\eta$ such that $x(z)=x^{\prime}+\eta z$ (paraxiality allows $\sin \eta \simeq \eta$, where $x^{\prime}$ is the original $x$-position), while the lower array is tilted by $-\eta$ (Fig. 1a). These two arrays combined exhibit different artificial GFs that cannot be gauged away by a coordinate transformation. The propagation of light in this structure is described by the paraxial wave equation

$$
i \partial_{z} \psi(\vec{r})=-\frac{1}{2 k_{0}} \nabla_{\perp}^{2} \psi(\vec{r})-\frac{k_{0}}{n_{0}} \Delta n(\vec{r}) \psi(\vec{r})
$$

Here, $\psi$ is the envelope of the electric field, $k_{0}$ is the optical wavenumber inside the bulk material, $n_{0}$ is the ambient refractive index, $\Delta n(\vec{r})=n(\vec{r})-n_{0}$ gives the relative refractive index profile, and $\nabla_{\perp}^{2}=\partial_{x}^{2}+\partial_{y}^{2}$. Eq. (1) is mathematically equivalent to the Schrödinger equation, where $z$ plays the role of time, and $\Delta n(\vec{r})$ plays the role of the potential. This analogy between the paraxial wave equation and the Schrödinger equation has been used many times in exploring a plethora of fundamental phenomena, ranging from Anderson localization $^{31}$ and Zener tunneling ${ }^{32}$ to non-Hermitian potentials $^{33}$ and Floquet topological insulators ${ }^{23}$.

The basic building block in our system is a twodimensional array of evanescently coupled straight waveguides, i.e., $\Delta n(\vec{r})$ is a periodic function in both $x$ and $y$, with periods $a_{x}$ and $a_{y}$, respectively, such that each unit cell consists of a single waveguide.

Consider first an array where the trajectories of all the waveguides are in the $z$-direction. Following coupled mode theory ${ }^{25}$, the spectrum of light propagating in such a $2 \mathrm{D}$ array of waveguides is given by

$$
\beta\left(k_{x}, k_{y}\right)=\beta_{0}+2 c_{x} \cos \left(k_{x} a_{x}\right)+2 c_{y} \cos \left(k_{y} a_{y}\right)
$$

where $\beta$ is the propagation constant of an eigenmode, defined by $\psi(x, y, z)=\psi_{0}(x, y) e^{-i \beta z}, k_{x}$ and $k_{y}$ are the spatial momenta of the mode in the $x$ - and $y$-directions, $c_{x}$ and $c_{y}$ are the coupling strengths between adjacent waveguides in the $x$ - and $y$-directions (taken to be real negative numbers according to standard solid state notation) and $\beta_{0}$ is the propagation constant of the guided mode in a single isolated waveguide. Consider now an array of waveguides tilted at an angle $\eta$ with respect to the $z$-axis such that the waveguides follow a trajectory defined by $x-\eta z=$ constant. The dynamics in an array of tilted waveguides are expressed by an artificial GF, given by the effective vector potential $\vec{A}(z)=-k_{0} \eta \hat{x}$, with the following spectrum ${ }^{25,27}$ :

$$
\begin{aligned}
\beta_{\eta}\left(k_{x}, k_{y}\right)= & \beta_{0}+2 c_{x} \cos \left(\left(k_{x}-k_{0} \eta\right) a_{x}\right)+\eta k_{x} \\
& -\frac{1}{2} k_{0} \eta^{2}+2 c_{y} \cos \left(k_{y} a_{y}\right)
\end{aligned}
$$

The shift of $k_{0} \eta$ in the cosine is the compensation due to the Galilean transformation of the waveguides. The linear $\eta k_{x}$ shift term appears because the spectrum in Eq. (3) is expressed in the laboratory frame and not in the frame of reference in which the waveguides are stationary. The constant offset $\frac{1}{2} k_{0} \eta^{2}$ results from the effective elongation of the optical path inside the tilted waveguides.

Such a linear tilt of a waveguide array is, in itself, a trivial gauge field, as we can eliminate its effects by changing the frame of reference to the co-moving frame, i.e., a linear coordinate transformation of the entire system can gauge it out. This can also be understood by examining the arising effective magnetic field $\vec{B}=\vec{\nabla} \times \vec{A}$, which is zero for a constant vector potential $\vec{A}$. To have a nontrivial gauge, we need the effective gauge field to be nonuniform (i.e., have either space or time dependence). Such a nontrivial gauge is achieved by coupling two 2D arrays, each with a different tilt angle and therefore a different gauge. Then, it becomes impossible to gauge away the effect of the tilt when we combine two such fields with different tilts. There is no coordinate system in which both arrays would be simultaneously untilted ${ }^{27}$. Here $\vec{A}=\eta k_{0} \widehat{x}$ at the upper section, and $\vec{A}=-\eta k_{0} \widehat{x}$ at the lower section.

With this in mind, consider a two-dimensional array of evanescently coupled waveguides divided into two regions -top and bottom, as shown in Fig. 1a. The rows of waveguides in the top and bottom regions are identical in every parameter except for the tilt. The overall GF is then given by

$$
\vec{A}(\vec{r})=(2 \Theta(y)-1) \eta k_{0} \hat{x}
$$



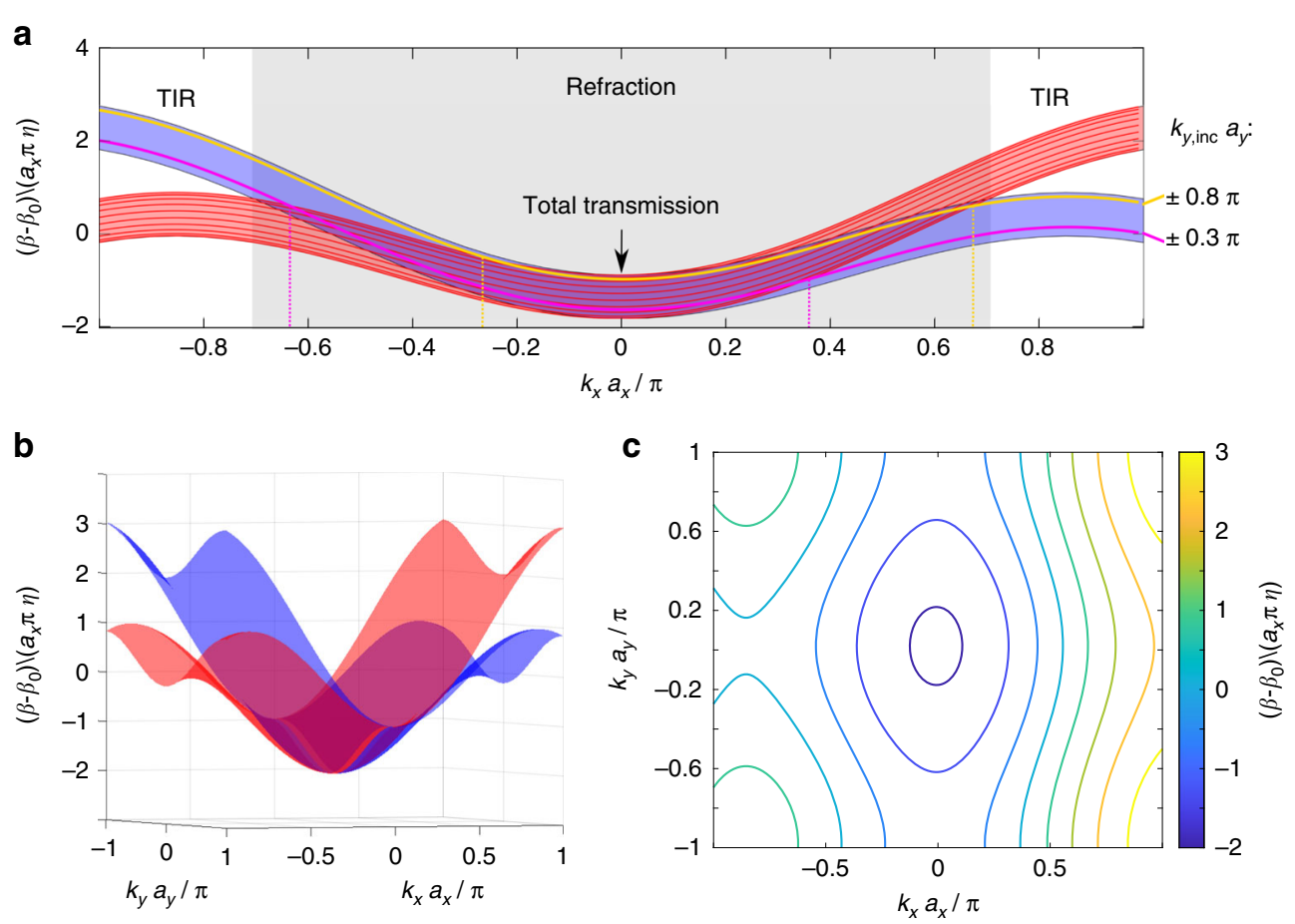

Fig. 2 Dispersion relations for the upper (red) and lower (blue) arrays. a Projection of $\beta$ as a function of $k_{x}$. The solid lines mark some specific values of $k_{y} a_{y}$. In the $k_{x}$-range where the two bands overlap, any wavepacket traveling across the artificial gauge interface undergoes refraction according to the generalized Snell law (shaded gray region). Outside this range, total internal reflection (TIR) occurs. Note that the $k_{x}$-range for total reflection (unshaded region) is not necessarily symmetric around $k_{x}=0$ (see vertical dashed lines, for a given $k_{y}$ ). The parameters here are the same as in the experiment: $r=0.52 \mu \mathrm{m}, a_{x}=1.69 \mu \mathrm{m}, a_{y}=2.15 \mu \mathrm{m}, \eta=0.0093$, and $\lambda=700 \mathrm{~nm}$. b Full 3D dispersion relation $\beta$ as a function of $k_{x}$ and $k_{y}$. c Contour plot of the equi- $\beta$ over $k_{x}$ and $k_{y}$ for the red band. Each line represents an equi- $\beta$ contour. The group velocity of a wavepacket at each point is perpendicular to the equi- $\beta$ contour that goes through that point

where $\Theta(y)$ is the Heaviside function, which is 1 when $y>$ 0 and zero otherwise. This vector potential gives an effective magnetic field $\vec{B}=-2 \delta(y) \eta k_{0} \hat{z}$ with a $\Phi_{B}=$ $-2 \eta k_{0} a_{x}$ magnetic flux through a unit cell at the interface.

The different gauge fields in each subsystem result in a different band structure (dispersion relation) for each subsystem. The system has discrete symmetries in both the $x$ - and $z$-directions, with periods of $a_{x}$ and $\frac{a_{x}}{2 \eta}$, respectively (see Supplementary Information). Each of these dictates a conservation law for the respective momentum (up to $2 \pi$ over the period), leaving only $k_{y}$ to be modified as a wave crosses between the two regions. Thus, launching an eigenmode (a Bloch wave) with a defined wavevector $\left(k_{x}, k_{y \text {,inc }}\right)$ on one side of the system will result in refraction and reflection of the wave upon incidence at the interface. According to the $x$ - and $z$ translational symmetries of the joint lattice, the wavenumber in the second half plane will have to satisfy

$$
\beta_{\eta}\left(k_{x}, k_{y, \text { inc }}\right)=\beta_{-\eta}\left(k_{x}, k_{y, \text { tran }}\right)
$$

where $k_{y \text {,inc }}$ is the $y$-wavenumber of the incident beam, $k_{y \text {,tran }}$ is the $y$-wavenumber of the transmitted beam, and $\beta_{\eta}\left(k_{x}, k_{y, \text { inc }}\right)$ is given by Eq. (3). Equation (5) acts as a generalized Snell law for an interface between two regions of the same medium but with different artificial gauge fields on each side in the specific realization of titled photonic lattices.

Note that Eq. (5) is general and valid for any interface that satisfies the symmetries in $x$ and $z$ (the plane normal to the interface), even for a uniform dielectric medium. The main difference between refraction from a dielectric interface and refraction from an AGF interface lies in the dispersion relation, that is, the relation between the propagation constant $\beta$ and the frequency. In uniform dielectrics, a plane wave (which is an eigenmode of the medium) obeys $\beta_{\text {dielectric }}=\sqrt{\left(\frac{n \omega}{c}\right)^{2}-\left(k_{x}^{2}+k_{y}^{2}\right)}$. On the other hand, for an AGF medium, the dispersion can be designed as almost any desired relation ${ }^{34,35}$. In the specific case of tilted waveguide arrays, the dispersion relation is given by Eq. (3). The ability to design the dispersion allows for interesting dynamics such as a negative group velocity for some $k_{x}$ values, for example, $2 c_{x} a_{x} \sin \left(\left(k_{x}-k_{0} \eta\right) a_{x}\right)<-\eta$ for the case of tilted waveguide arrays. Engineering the dispersion relation also makes it possible to cancel diffraction in one of the 


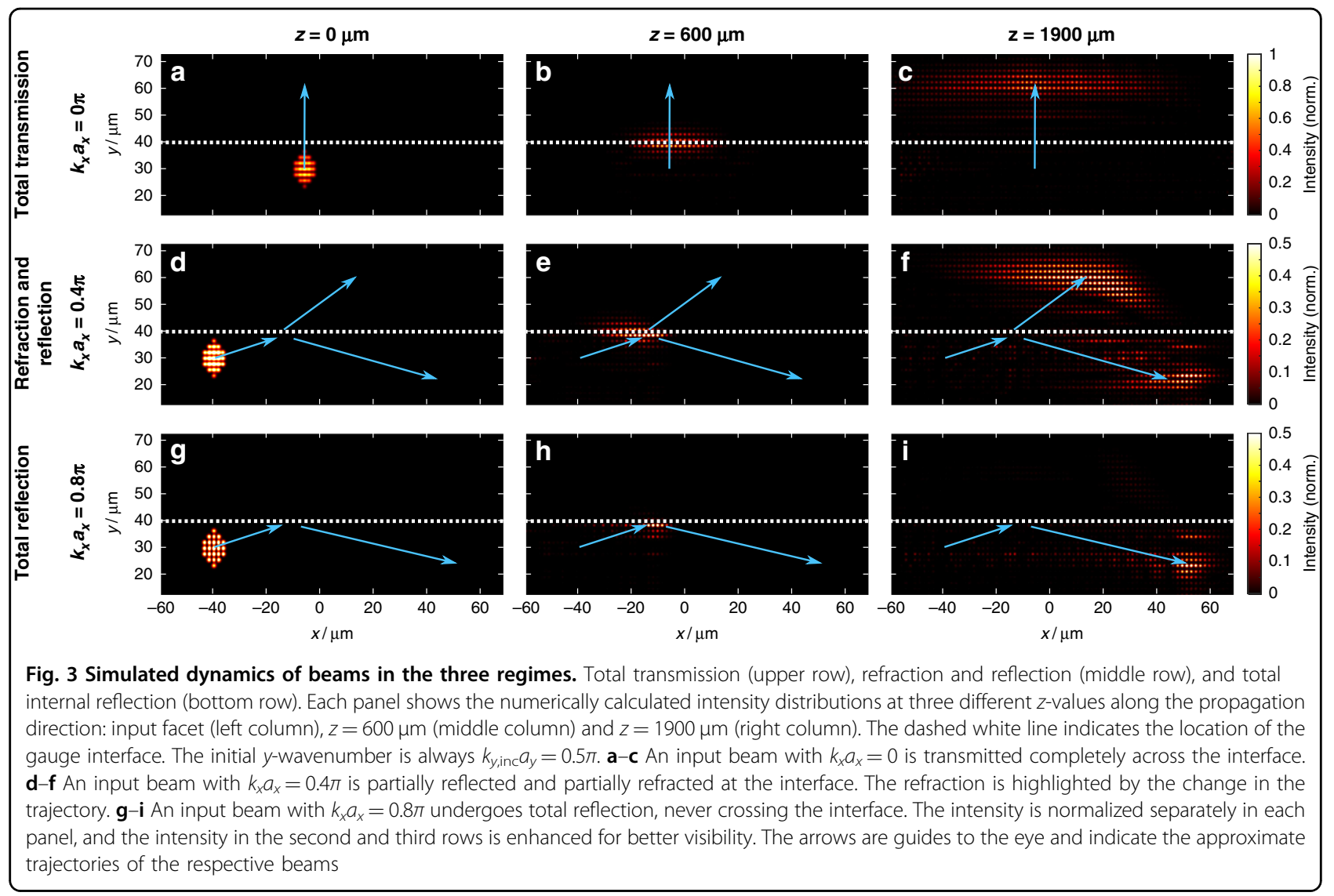

directions, as we suggest in the "Discussion" section (Fig. 7). Notably, uniform dielectric interfaces require materials with different optical properties on each side of the interface, whereas an AGF interface can be engineered even when both sides have the same optical properties (up to their gauge), achieving refraction using the same materials of the same composition and configuration (periodicity).

Figure 2 shows the band structures for the upper (red) and lower (blue) waveguide arrays. Depicted in three dimensions (Fig. 2b), we note the sinusoidal shape of the dispersion along $k_{x}$ as well as along $k_{y}$. The projection onto the $k_{x}$-component (Fig. 2a), however, helps us display the $k_{y}$-conversion between the two arrays. In the projected band structure, each band represents the values of $\beta$ (which plays the role of energy in the analogy to the Schrödinger equation) for all values of $k_{y}$ associated with that band (see the Supplementary Information for a discussion on band replicas arising from the periodicity of the structure in $x$ and $z$ ). As an example, the solid lines in Fig. $2 \mathrm{a}$ indicate the values of $\beta$ associated with some specific $k_{y}$. Note that the $k_{x}$-range for total reflection (dashed vertical lines in Fig. 2a) is not necessarily symmetric around $k_{x}=0$ (for a given $k_{y}$ ). From this figure, it may seem that $\beta$ is not periodic in $k_{x}$, but a closer look at the symmetries in the system reveals that the periodicity is maintained (see the discussion in the Supplementary Information). Figure $2 c$ displays a contour plot of equi- $\beta$ as a function of $k_{x}$ and $k_{y}$. The group velocity of a wavepacket at each point is perpendicular to the equi- $\beta$ contour that goes through that point.

When a wavepacket crosses the artificial GF interface between the lower array and the upper array, $\beta$ is conserved such that $\beta_{\eta}\left(k_{x}, k_{y \text {,inc }}\right)=\beta_{-\eta}\left(k_{x}, k_{y, \text { tran }}\right)$, as is the transverse wavenumber, $k_{x, \text { inc }}=k_{x, \text { tran }}=k_{x}$. Graphically, this means that at this value of $\beta$ the red and blue bands in Fig. 2a overlap. The quasi-energy $\beta$ of the red band may belong to a different $k_{y}$ than that of the blue band; thus, $k_{y \text {,inc }} \neq k_{y, \text { tran }}$ (see solid colored lines in Fig. 2a). Therefore, when the light crosses the artificial GF interface, $k_{\mathrm{y}, \text { inc }}$ must change according to

$$
\begin{aligned}
& \cos \left(k_{y, \text { tran }} a_{y}\right)-\cos \left(k_{y, \text { inc }} a_{y}\right) \\
& =\frac{c_{x}}{c_{y}}\left[\cos \left(\left(k_{x}+k_{0} \eta\right) a_{x}\right)-\cos \left(\left(k_{x}-k_{0} \eta\right) a_{x}\right)\right]-\eta \frac{k_{x}}{c_{y}}
\end{aligned}
$$

We identify three different regimes, which depend on $k_{x}$ : 

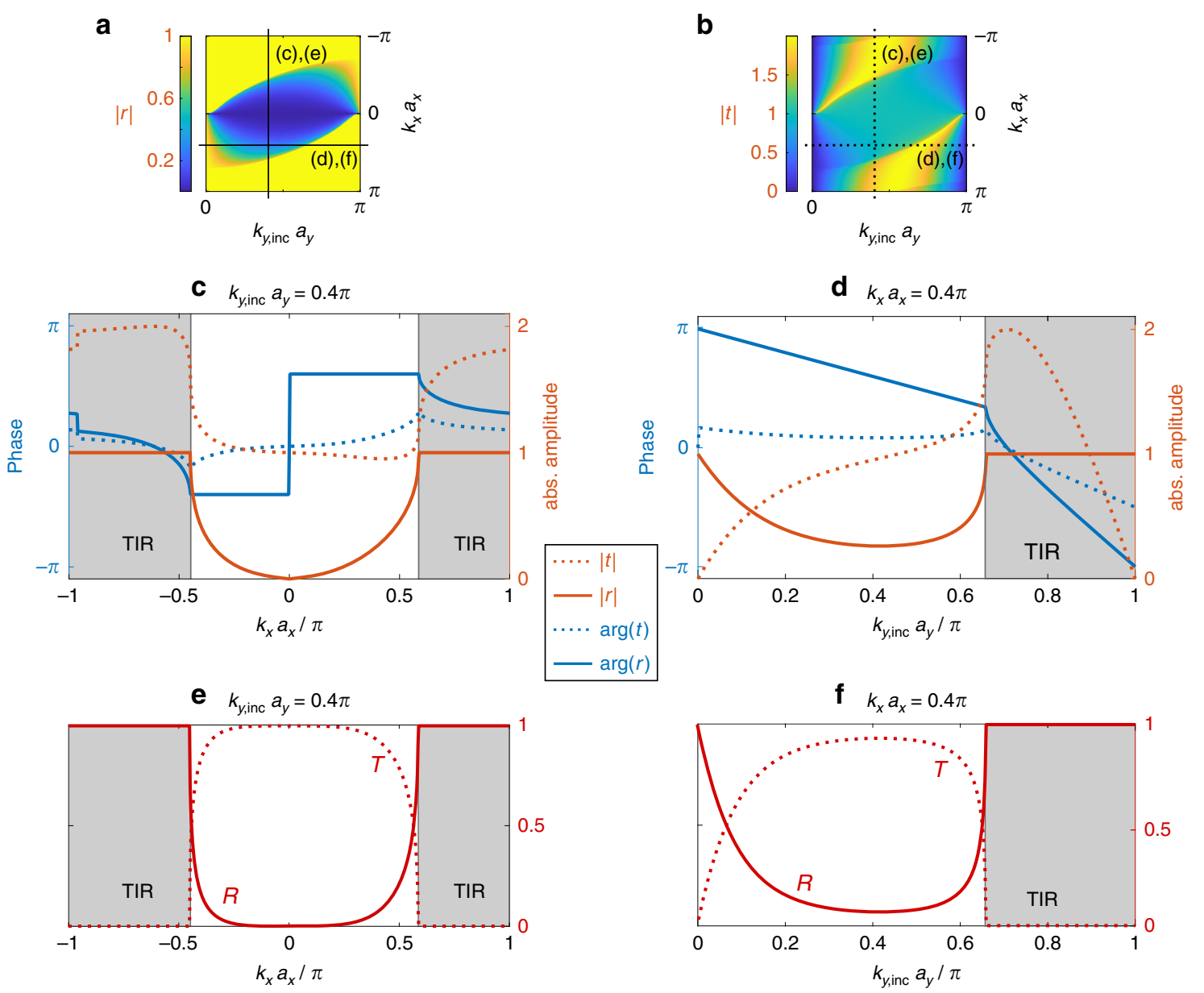

Fig. 4 Fresnel coefficients. Reflection amplitude (a) and transmission amplitude (b) across the entire $k_{x}$ and $k_{y}$ range. The line cuts in the middle row show the phase (blue lines) and amplitude (red lines) of the reflection coefficient $r$ (solid lines) and the transmission coefficient $t$ (dashed lines) along the cuts indicated in (a) and (b): for $k_{y, i n c} a_{y}=0.4 \pi(\mathbf{c})$ and $k_{x} a_{x}=0.4 \pi(\mathbf{d})$. Note that $|t|$ can be greater than 1 for some $\left(k_{x}, k_{y, \text { inc }}\right.$ ). e and $\mathbf{f}$ show the reflectance $R=|r|^{2}$ (solid line) and transmittance $T=\operatorname{Re}\left\{\frac{v_{\text {gyy tran }}}{V_{\text {gy,inc }}}\right\}|t|^{2}$ (dashed line) for the same wave vectors as in (c) and (d). The total energy flux is conserved, as $R+T=1$ holds for all regions

1. Total internal reflection (TIR): $k_{x}$ is such that the blue and red bands do not intersect, hence no coupling from the upper to the lower array (and vice versa) is possible. Consequently, the wavepacket is completely reflected (see Fig. 3g-i).

2. Perfect transmission for $k_{x}=0$ : A wavepacket crosses the interface between the two arrays without changing its wave vector components while allowing all the light to be transmitted through the interface (see Fig. 3a-c).

3. Refraction and reflection for all other values of $k_{x}$ : The red and blue bands intersect, but for different $k_{y}$, inc and $k_{y, \text { tran. }}$. As the "energy" $\beta$ and the transverse wavenumber $k_{x}$ are conserved, $k_{y}$ has to change upon crossing the interface, resulting in both a refracted wave and a reflected wave (Fig. $3 \mathrm{~d}-\mathrm{f}$ ).
Examples of the dynamics of waves in these three regimes are given in Fig. 3, which shows the results of direct simulations of Eq. (1) (using the commercial OptiBPM code), with parameters corresponding to those used in the experiments. The figure shows the intensity of optical beams at three different propagation planes along $z$. We probe the three different regimes by launching input beams with a set $k_{y \text {,inc }} a_{y}=0.5 \pi$ and selecting $k_{x} a_{x}$ corresponding to total transmission $\left(k_{x} a_{x}=0\right)$, refraction and reflection $\left(k_{x} a_{x}=0.4 \pi\right)$, and total internal reflection $\left(k_{x} a_{x}=0.8 \pi\right)$. Upon excitation at $z=0 \mu \mathrm{m}$ (Fig. 3a, d, g), the beams travel toward the interface (indicated by the dashed white line), reaching it approximately after $z=$ $600 \mu \mathrm{m}$ (Fig. 3b, e, h). For the input beam with $k_{x} a_{x}=0$, the beam is completely transmitted across the interface (Fig. 3c) without any reflection. After passing through the 


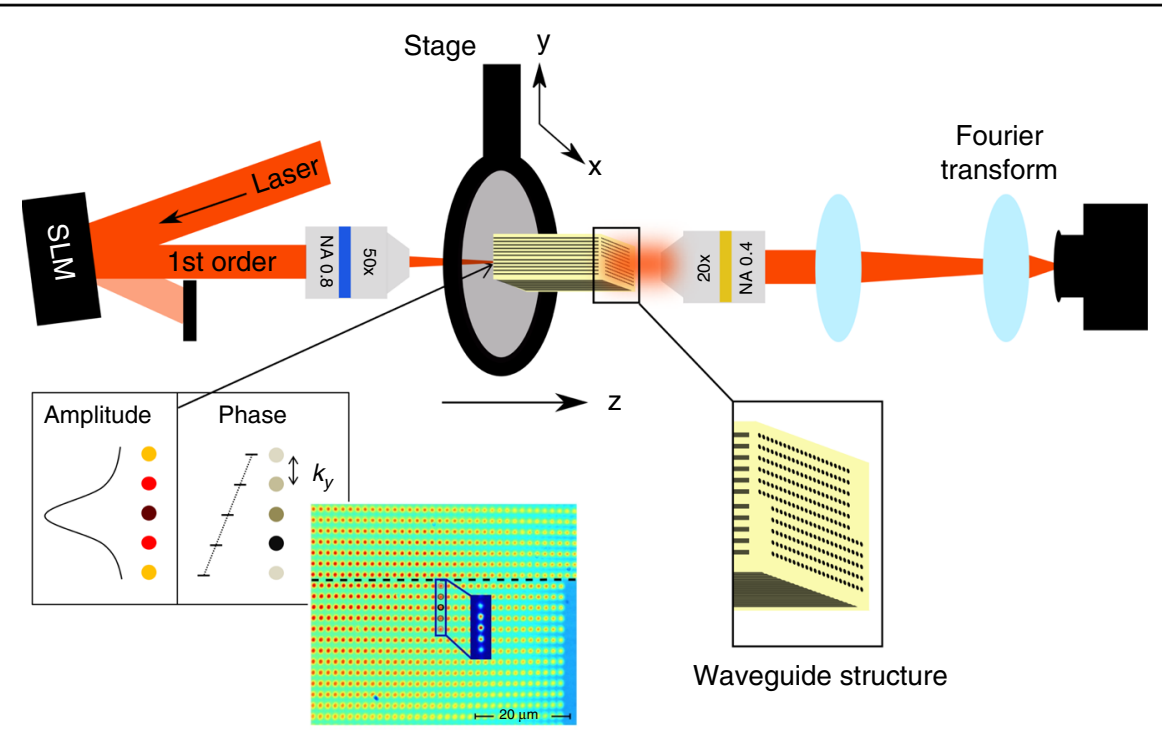

Fig. 5 Experimental setup. A laser beam (wavelength 700nm) is reflected off an SLM, which imprints a specific phase and amplitude pattern onto the beam. To shape the amplitude while using a phase-only SLM, we overlay the phase pattern on the SLM with a blazed grating that shifts parts of the reflected light into the first diffraction order ${ }^{37}$. After Fourier transform by an objective, the beam consists of five spots with a phase difference of $k_{y, \text { inc }} a_{y}$ between each of them and a Gaussian amplitude envelope (inset). These spots are focused onto a row of waveguides below the interface, as shown by the false color photograph of the beam on top of the sample, with the interface marked by the dashed line. The light then propagates along $z$, interacts with the interface, and exits the sample after a propagation distance of $z=725 \mu \mathrm{m}$. The intensity distribution at the output facet is imaged by a camera, as is its spatial power spectrum (obtained by inserting an extra lens at the focal distance to the camera)

interface, the beam strongly disperses (diffracts) in the $x$ direction. For the input beam with $k_{x} a_{x}=0.4 \pi$, part of the beam is reflected by the interface, returning to the lower array, while part of it is refracted, as indicated by the change in the slope of the arrow (Fig. 3f). For the beam with $k_{x} a_{x}=0.8 \pi$, the beam undergoes total reflection, never crossing the interface (Fig. 3i).

Having used symmetry and the dispersion relation to find the general laws of refraction and reflection at a gauge interface, the next step is natural: finding the coefficients for reflection and transmission. However, similar to the Fresnel coefficients at a dielectric interface, this calculation is system specific, and the details depend on the interface between the two regions. That is, unlike the Snell-like law, the Fresnel coefficients cannot be generalized (conservation of power yields a relation between the absolute values of the Fresnel coefficients, but to obtain the coefficients, one must also employ continuity at the interface). With this in mind, we calculate the Fresnel-like coefficients for our example of a gauge interface constructed from titled waveguides. We use an approximate model ${ }^{27}$ for the coupling between the two sections and derive an approximate formula for the coefficients. The details of the calculation are presented in the Supplementary Information. Figure 4 shows the Fresnel-like coefficients for our gauge interface for parameters corresponding to those shown in Fig. 3. We plot the amplitude and phase of the transmitted and reflected parts for $k_{y, \text { inc }} a_{y}=0.4 \pi$ as a function of $k_{x}$ and for $k_{x} a_{x}=0.4 \pi$ as a function of $k_{y, \text { inc }}$ (Fig. $4 \mathrm{c}$, d, respectively). As explained above, the Fresnel coefficients are highly dependent on the specifics of the interface; hence, a different model for the gauge field interface will yield different coefficients. However, the Snell-like law of refraction will not change, as it depends only on the symmetry and the dispersion relations on both sides of the interface.

To demonstrate the generalized Snell's law in experiments, we fabricate sets of tilted optical waveguides corresponding to the system described in Fig. 1. The samples are fabricated using direct laser writing to create hollow waveguides, which are subsequently infiltrated with a higher index material (Fig. 1c, d). For details on the fabrication, see ref. ${ }^{36}$. The experimental measurement setup is sketched in Fig. 5. We reflect a $700 \mathrm{~nm}$ laser beam off a spatial light modulator (SLM) to excite a Bloch mode with a given $k_{y, \text { inc }}$ while exciting the entire first Brillouin zone in $x$ (i.e., $-\pi \leq k_{x} a_{x} \leq \pi$ ). To do this, the beam reflected off the $\mathrm{SLM}^{37}$ is shaped such that after a Fourier transform (by a microscope objective), it consists of 5 lobes, with their phase forming a linear ladder, commensurate with the chosen Bloch mode, while in $x$, the beam is simply focused into a single row of waveguides. The beam passes through the sample, and the output facet is imaged by a camera. Since we are interested in investigating the passage through the gauge interface, we scan the value of 


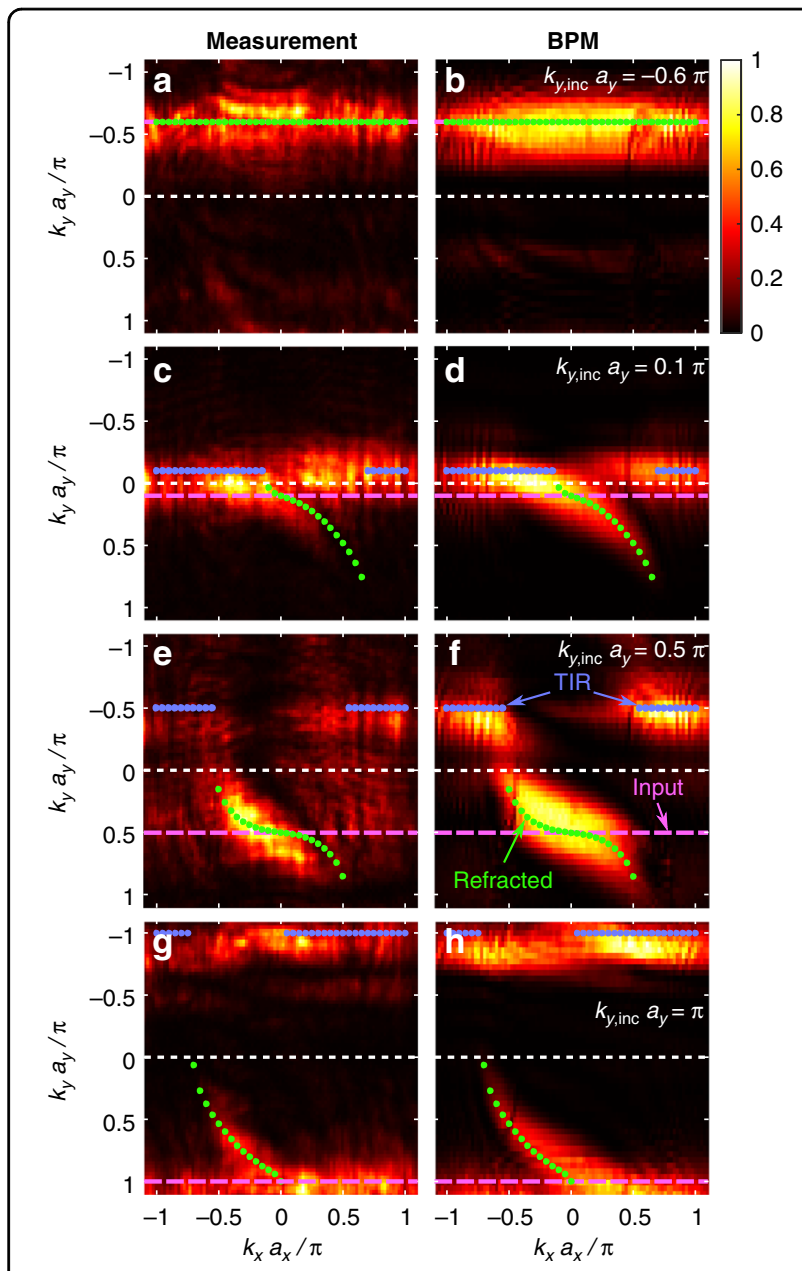

Fig. 6 Refraction and reflection by an artificial gauge interface. Spatial power spectrum (intensity in Fourier space) for an input wave with several values of $k_{y, \text { inc }}$ always launched at the same position in $y$. The left and right columns depict the experimental and simulated results, respectively. The purple dashed line shows the location of the input beam in Fourier space and the dots show the values analytically calculated from Eq. (6) (green for refraction and blue for TIR). For $k_{y, i n c}$ $a_{y}=-0.6 \pi$, the beam travels away from the interface and does not refract at all $(\mathbf{a}, \mathbf{b})$, while for the other panels $(\mathbf{c}-\mathbf{h})$, we see both partial refraction and reflection. In the second row $(\mathbf{c}, \mathbf{d})$, the beam is

launched with $k_{y} a_{y}=0.1 \pi$. As these panels show, the output beam is split: for $-0.1 \pi<k_{x} a_{x}<0.6 \pi$, the beam is transmitted and displays

distinct refraction, according to the generalized Snell's law expressed by Eq. (6), while in the regions beyond this range, the beam

experiences TIR. Note the prominent asymmetry between the minimal and maximal $k_{x}$ boundaries between the regions of transmission and TIR. In the third row $(\mathbf{e}, \mathbf{f})$, the beam is launched with $k_{y} a_{y}=0.5 \pi$. For $-0.5 \pi<k_{x} a_{x}<0.5 \pi$, the beam is transmitted, while beyond this range, the beam experiences TIR. The experiment (e) captures both the refraction and TIR regions. In the 4th row, $(\mathbf{g}, \mathbf{h})$, the beam is launched with $k_{y} a_{y}=\pi$. For $-0.7 \pi<k_{x} a_{x}<0.1 \pi$, the beam is transmitted. Beyond this domain, the beam experiences TIR. Here, both the incident and reflected beams have the same $\left|k_{y}\right|$. Altogether, the comparison of the measurements (left row), simulations (right row), and analytical expression from Eq. (6) (green and blue dots) shows good agreement with the expected $k_{y, t r a n}$-distribution. A movie showing the complete set of measurements can be found in Supplementary Movie no. \#1. $k_{y, \text { inc }}$ by changing the relative phase between the lobes while exciting the entire first Brillouin zone in $x$. Figure 5 shows a false color photograph of the input beam overlay a photograph of the sample, with the interface marked by the dashed line. The light propagates across the interface, and we measure the intensity of the refracted and reflected waves at the output facet of the sample, as well as the intensity at the Fourier plane (obtained at the focal plane of a lens), which corresponds to the spatial power spectrum.

Figure 6 shows the spatial power spectrum (Fourier space intensity) of the waves exiting the sample for an input wave with different $k_{y \text {,inc }}$ values but always launched at the same position in $y$, along with a comparison to beam-propagation simulations. The analytically calculated values for $k_{y, \text { tran }}$ obtained by Eq. (6) are marked by the green and blue dots on top of the experimental and simulated results. The beam travels toward the artificial GF interface with a group velocity $v_{g y}$, obtained from the dispersion relation in Eq. (3) by taking the derivative with respect to $k_{y} a_{y}$. In the first row (Fig. 6a, b), the beam is launched such that it moves away from the interface $\left(k_{y} a_{y}=\right.$ $-0.6 \pi)$, and never reaches the interface; hence, the output beam has the same spatial spectrum as the input beam. As Fig. 6a, b show, the power spectrum of the output beam is located around the same $k_{y} a_{y}=-0.6 \pi$ as the input beam. In the second row (Fig. $6 \mathrm{c}, \mathrm{d}$ ), the beam is launched with $k_{y} a_{y}=0.1 \pi$. As these panels show, the output beam is split: for $-0.1 \pi<k_{x} a_{x}<0.6 \pi$, the beam is transmitted and displays distinct refraction, according to the generalized Snell's law expressed by Eq. (6), while in the regions beyond this range, the beam experiences TIR. Note the prominent asymmetry between the minimal and maximal $k_{x}$ boundaries between the regions of transmission and TIR. In experiment (c), the measurement only partially shows the results, as the group velocity in $y$ for $k_{y} a_{y}=0.1 \pi$ is very low, and the beam has only partially passed the interface even upon reaching the output facet of the sample. In the third row, Fig. 6e, $\mathrm{f}$, the beam is launched with $k_{y} a_{y}=0.5 \pi$. For $-0.5 \pi<k_{x} a_{x}<0.5 \pi$, the beam is transmitted, while beyond this range, the beam experiences TIR. Here, the asymmetry between the minimal and maximal $k_{x}$ boundaries between transmission and TIR is less significant. The experiment (Fig. 6e) captures both the refraction and TIR regions. In the fourth row, Fig. $6 \mathrm{~g}$, $\mathrm{h}$, the beam is launched with $k_{y} a_{y}=\pi$. For $-0.7 \pi<k_{x} a_{x}<$ $-0.1 \pi$, the beam is transmitted. Beyond this domain, the beam experiences TIR. Here, both the incident and reflected beams have the same $\left|k_{y}\right|$. This case is similar to prism coupling at a grazing angle to couple a beam into a waveguide where it will be bound by TIR.

The comparison of the experimental measurements and numerical calculations with the analytical Eq. (6) (dots in Fig. 6) shows good agreement for the refracted part. 

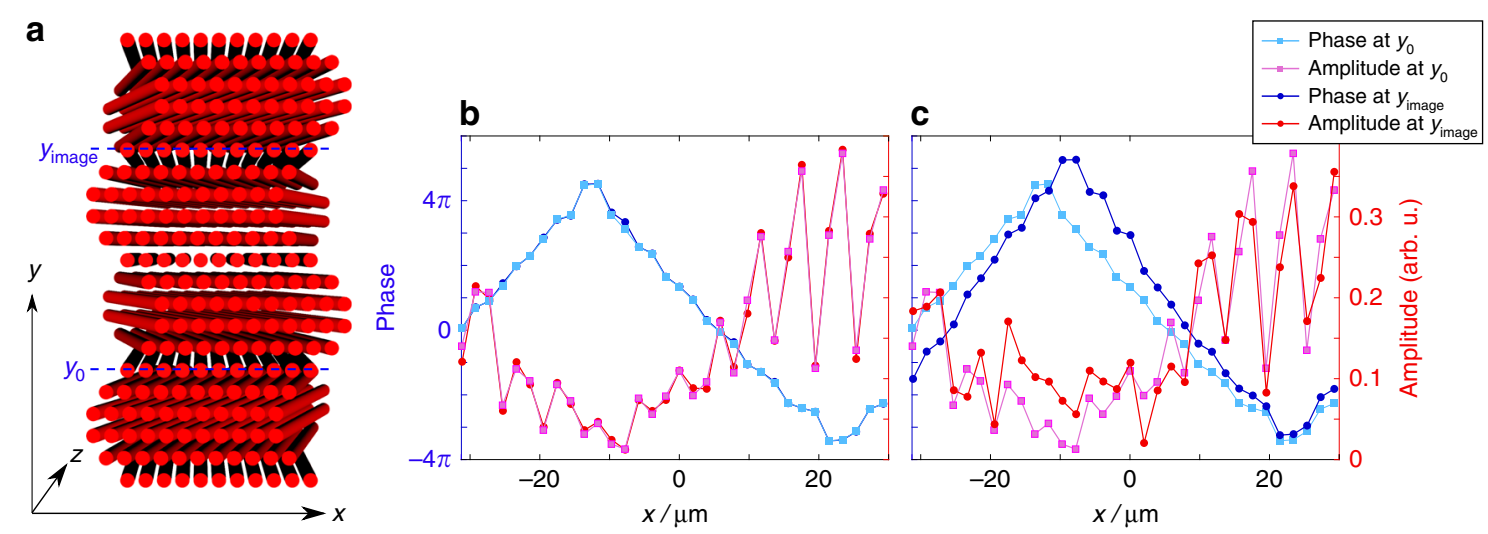

Fig. 7 Gauge-based imaging system. a Sketch of a gauge-based imaging system. The tilt angle $\eta$ for each row as a function of $y$ is given by $\eta(y)=\eta_{0} \sin \left(\frac{2 \pi\left(y-y_{0}\right)}{y_{\text {image }}-y_{0}}\right)$. We launch the beam at $y_{0}$ and expect it to reconstruct at $y_{\text {image. }}$ In the calculations, we assume a periodicity in $x$ of 32 waveguides. b, c The amplitude (pink) and phase (light blue) at $y=y_{0}$ and $z=0$ (squares) compared to the amplitude (red) and phase (dark blue) at $y=y_{\text {image }}$ (circles). $\mathbf{b}$ The system parameters satisfy Eq. (8), and reconstruction in both the amplitude and phase is achieved. The output distance $z$ is chosen such that the maximal total power is obtained at the image plane (which occurs at $z=1000 \mu \mathrm{m}$ ). c The system parameters are the same as in (b) except for $\eta_{0}$ such that now, it does not satisfy Eq. (8). Even at the $z$ with the best fit by cross-correlation $(z=926 \mu$ m), the original signal differs completely from the output signal

As expected, the obtained $k$-distribution is broader than the analytical curve due to finite size effects. Namely, the input beam with $k_{y \text {,inc }}$ has a finite width (see Fig. 5) of five waveguides. The more waveguides are excited in real space, the smaller the width of the $k$-component in Fourier space. However, we do not want the input pattern to excite waveguides in the other array across the artificial GF interface; hence, we have to limit the size of the input beam. In addition, the center position of the input pattern needs to be close enough to the artificial GF interface such that the beam can travel across the artificial GF interface in the given propagation distance. Therefore, we need to limit the number of excited waveguides. For excitation of five waveguides, the width is $\Delta k_{y \text {,inc }} a_{y} \approx 0.4 \pi$ (see Fig. 6). As the same number of illumination spots is chosen in the experiments, the numerical calculation reflects the experimental conditions very well. Altogether, the comparison of the measurements, simulations, and analytical expression shows good agreement with the expected $k_{y}$, tran-distribution. A movie showing the complete set of measurements can be found in Supplementary Movie \#1.

\section{Discussion}

Having demonstrated the Snell law for refraction and reflection at an interface between two different artificial GFs, we move on to concatenating several gauge interfaces and constructing devices. As an example highlighting the possibilities that refraction by artificial GFs allows, we design a gauge-based imaging system. By realizing such a system with arrays of tilted waveguides, we design a scheme that maps any (arbitrary) wavepacket input at the input facet to the output facet. In the scheme based on waveguide arrays, this corresponds to a system with different rows of waveguides tilted at different angles (Fig. 7a) mapping an input state from row $y_{0}$ to row $y_{\text {image. }}$ For every row of waveguides with a tilt $\eta(y)$ positioned at $y$, we find the propagation constant $\beta_{\eta(y)}=\beta_{0}+$ $2 c_{x} \cos \left(\left(k_{x}-k_{0} \eta(y)\right) a_{x}\right)+\eta(y) k_{x}-\frac{1}{2} k_{0} \eta^{2}(y)$. To produce an image, we need the phase accumulation by all components to be identical. Thus, we require that when a wavepacket diffracts along $y$ and propagate along $z$ from input row $y_{0}$ to output row $y_{\text {image }}$, the cumulative phase accumulation for each of its $k_{x}$ constituents is the same. Therefore, the value of $\int_{0}^{y_{\text {image }}} \beta_{\eta(y)}\left(k_{x}\right) \mathrm{d} y$ should not depend on $k_{x}$. This can be written as

$$
\frac{\mathrm{d}}{\mathrm{d}\left(k_{x}\right)} \int_{0}^{y_{\text {image }}} \beta_{\eta(y)}\left(k_{x}\right) \mathrm{d} y=0
$$

This requirement can be fulfilled in a 2D array of straight waveguides, where each row $(y)$ is tilted at a different angle such that $\eta(y)=\eta_{0} \sin \left(\frac{2 \pi y}{y_{\text {image }}}\right)$. The requirement in Eq. (7) can be expressed by

$$
\int_{0}^{2 \pi} \exp \left(-i k_{0} \eta_{0} a_{x} \sin \left(y^{\prime}\right)\right) \mathrm{d} y^{\prime}=J_{0}\left(k_{0} \eta_{0} a_{x}\right)=0
$$

where $J_{0}$ is the zeroth-order Bessel function and $y^{\prime}=\frac{2 \pi y}{y_{\text {image }}}$ is now unitless. In other words, by designing the tilt of each row properly, an arbitrary wavepacket $f(x)$ at row $y=$ 0 is reproduced at row $y_{\text {image }}$ (apart from a global phase). Figure 7a shows a sketch of the gauge-imaging waveguide structure. Each row has a different tilt angle $\eta(y)=\eta_{0} \sin \left(\frac{2 \pi\left(y-y_{0}\right)}{y_{\text {image }}-y_{0}}\right)$. Figure $7 \mathrm{~b}$ compares the amplitude 
and phase of the wavepacket launched at $y=y_{0}$ and $z=0$ (pink and light blue) to those of the imaged one at $y=$ $y_{\text {image }}$ and $z=1000 \mu \mathrm{m}$ (red and dark blue), revealing that the final and initial wavepackets are essentially the same. One should note that the beam diffracts in $y$ as it propagates along $z$; hence, the imaging is one-dimensional for the field distribution in $x$ only. Therefore, the intensity reaching the row at $y_{\text {image }}$ is limited by the $1 \mathrm{D}$ discrete diffraction in $z$ such that the intensity at each row (neglecting back reflections) is given approximately by $\left|J_{\frac{\Delta y}{a_{y}}}(f(z))\right|^{2}$, where $f(z)$ is a function of $z$ that depends on the details of the coupling and $J_{\frac{\Delta y}{y}}$ is the Bessel function of the order of row number $\frac{\Delta y}{a_{y}}$. In our simulated example, there are 29 rows of waveguides between $y_{0}$ and $y_{\text {image }}$, so the maximal intensity that can propagate to $y_{\text {image }}$ is limited to $\max \left|J_{29}\right|^{2} \approx 4.7 \%$ of the initial intensity. In practice, we obtain approximately $2.9 \%$ due to back reflections (in $y$ ) and slightly different effective couplings along $y$ for each $k_{x}$ component. In the simulation, we assume that the structure is periodic in $x$ with a period of 32 waveguides. The output facet is chosen to support maximal total power at the imaging row. Figure $7 \mathrm{c}$ uses the same system as Fig. $7 \mathrm{~b}$, changing only the size of $\eta_{0}$ such that Eq. (8) is no longer fulfilled. We simulate the propagation of the same wavepacket in this (non-imaging) structure up to the distance that gives the maximal crosscorrelation between the input and the output, which occurs at $z=926 \mu \mathrm{m}$, yet it is clear that the input and output wavepackets do not overlap. Essentially, with proper design of this simple gauge-based imaging system, we can transfer an arbitrary wavepacket from an initial row at the input facet of the structure to a preselected row at the output facet. This idea works well as long as the Bloch mode spectrum in $x$ never projects onto evanescent modes (generated by TIR) throughout the propagation. This example, albeit simple, demonstrates that it is possible to construct various optical devices and systems by engineering artificial gauge fields using just one dielectric material.

To summarize, we derived the laws of refraction and reflection at an interface between two regions differing solely by their artificial gauge fields and demonstrated the concepts in experiments in 3D-micro-printed optical waveguide arrays. Generalizing the concepts of refraction and reflection at a gauge interface offers exciting possibilities for routing light and more generally for constructing photonic systems in a given medium strictly by designing the local gauge. As an example, we proposed an imaging system that maps any input state from one place at the input facet to a predesignated other location at the output facet by cascading different artificial gauge fields.

\section{Acknowledgements}

G.v.F. acknowledges support by the Deutsche Forschungsgemeinschaft through CRC/Transregio 185 OSCAR (project No. 277625399). M.S. gratefully acknowledges support by an ERC Advanced Grant, by the Israel Science Foundation, and by the German-Israel DIP project.

\section{Author details}

'Physics Department, Technion—Israel Institute of Technology, Haifa 32000, Israel. ${ }^{2}$ Solid State Institute, Technion-Israel Institute of Technology, Haifa 32000, Israel. ${ }^{3}$ Physics Department and Research Center OPTIMAS, TU Kaiserslautern, 67663 Kaiserslautern, Germany. ${ }^{4}$ Department of Physics, The Pennsylvania State University, State College, PA 16802, USA. ${ }^{5}$ Fraunhofer Institute for Industrial Mathematics ITWM, 67663 Kaiserslautern, Germany

\section{Author contributions}

All authors contributed significantly to this work.

\section{Data availability}

All experimental data and any related experimental background information not mentioned in the text are available from the authors upon reasonable request.

\section{Conflict of interest}

The authors declare that they have no conflict of interest.

Supplementary information is available for this paper at https://doi.org/ 10.1038/s41377-020-00411-7.

Received: 28 May 2020 Revised: 14 September 2020 Accepted: 14 September 2020

Published online: 22 December 2020

\section{References}

1. Snyder, A. W. \& Love, J. Optical Waveguide Theory. (Springer: Berlin, 2012).

2. Joannopoulos, J. D. et al. Photonic Crystals: Molding the Flow of Light. 2nd edn. (Princeton University Press, Princeton, 2008).

3. Segev, M., Silberberg, Y. \& Christodoulides, D. N. Anderson localization of light. Nat. Photonics 7, 197-204 (2013).

4. Veselago, V. G. The electrodynamics of substances with simultaneously negative values of $\varepsilon$ and $\mu$. Sov. Phys. Uspekhi 10, 509-514 (1968).

5. Pendry, J. B. Negative refraction makes a perfect lens. Phys. Rev. Lett. 85, 3966-3969 (2000)

6. Tamm, I. Über eine mögliche art der elektronenbindung an kristalloberflächen Z. Phys. 76, 849-850 (1932).

7. Shockley, W. On the surface states associated with a periodic potential. Phys. Rev. 56, 317-323 (1939).

8. Ritchie, R. H. Plasma losses by fast electrons in thin films. Phys. Rev. 106, 874-881 (1957).

9. Barnes, W. L., Dereux, A. \& Ebbesen, T. W. Surface plasmon subwavelength optics. Nature 424, 824-830 (2003).

10. D'yakonov, M. I. New type of electromagnetic wave propagating at an interface. Z. Eksp. Teor. Fiz. 11, 119-123 (1988).

11. Takayama, O. et al. Observation of Dyakonov surface waves. Phys. Rev. Lett. 102, 043903 (2009)

12. Kane, C. L. \& Mele, E. J. Quantum spin hall effect in graphene. Phys. Rev. Lett. 95 226801 (2005).

13. König, M. et al. Quantum spin hall insulator state in $\mathrm{HgTe}$ quantum wells. Science 318, 766-770 (2007).

14. Hsieh, D. et al. A topological Dirac insulator in a quantum spin Hall phase Nature 452, 970-974 (2008).

15. Szameit, A. et al. Fresnel's laws in discrete optical media. N. J. Phys. 10, 103020 (2008).

16. Umucalliar, R. O. \& Carusotto, I. Artificial gauge field for photons in coupled cavity arrays. Phys. Rev. A 84, 043804 (2011).

17. Hafezi, M. et al. Robust optical delay lines with topological protection. Nat. Phys. 7, 907-912 (2011).

18. Fang, K. J., Yu, Z. F. \& Fan, S. H. Realizing effective magnetic field for photons by controlling the phase of dynamic modulation. Nat. Photonics 6, 782-787 (2012). 
19. Rechtsman, M. C. et al. Strain-induced pseudomagnetic field and photonic Landau levels in dielectric structures. Nat. Photonics 7 153-158 (2013).

20. Madison, K. W. et al. Vortex formation in a stirred bose-einstein condensate. Phys. Rev. Lett. 84, 806-809 (2000).

21. Lin, Y. J. et al. Synthetic magnetic fields for ultracold neutral atoms. Nature $\mathbf{4 6 2}$ 628-632 (2009).

22. Bernevig, B. A. \& Hughes, T. L. Topological Insulators and Topological Superconductors. (Princeton University Press, Princeton, 2013).

23. Rechtsman, M. C. et al. Photonic Floquet topological insulators. Nature 496, 196-200 (2013).

24. Hafezi, M. et al. Imaging topological edge states in silicon photonics. Nat. Photonics 7, 1001-1005 (2013).

25. Plotnik, Y. et al. Analogue of Rashba pseudo-spin-orbit coupling in photonic lattices by gauge field engineering. Phys. Rev. B 94, 020301 (2016).

26. Lin, Q. \& Fan, S. H. Light guiding by effective gauge field for photons. Phys. Rev. $X$ 4, 031031 (2014).

27. Lumer, Y. et al. Light guiding by artificial gauge fields. Nat. Photonics 13, 339-345 (2019).

28. Wu, L. H. \& Hu, X. Scheme for achieving a topological photonic crystal by using dielectric material. Phys. Rev. Lett. 114, 223901 (2015).
29. Shalaev, M. I. et al. Robust topologically protected transport in photonic crystals at telecommunication wavelengths. Nat. Nanotechnol. 14, 31-34 (2019).

30. Fang, K. J. \& Fan, S. H. Controlling the flow of light using the inhomogeneous effective gauge field that emerges from dynamic modulation. Phys. Rev. Lett. 111, 203901 (2013).

31. Schwartz, T. et al. Transport and Anderson localization in disordered twodimensional photonic lattices. Nature 446, 52-55 (2007).

32. Dreisow, F. et al. Bloch-zener oscillations in binary superlattices. Phys. Rev. Lett. 102, 076802 (2009).

33. Makris, K. G. et al. Beam dynamics in PT symmetric optical lattices. Phys. Rev Lett. 100, 103904 (2008).

34. Sheinfux, H. H. et al. Recasting Hamiltonians with gauged-driving. 2017 Conference on Lasers and Electro-Optics (CLEO) (OSA, San Jose, 2017).

35. Rahav, S., Gilary, I. \& Fishman, S. Effective Hamiltonians for periodically driven systems. Phys. Rev. A 68, 013820 (2003).

36. Fedorova (Cherpakova), Z. et al. Limits of topological protection under local periodic driving. Light Sci. Appl. 8, 63 (2019).

37. Waller, E. H., Renner, M. \& von Freymann, G. Active aberration- and pointspread-function control in direct laser writing. Opt. Express 20, 24949-24956 (2012). 